\section{The best crude timer for MS-DOS implementations of $\mathbf{C}$}

\section{PHILLIP L. EMERSON \\ Cleveland State University, Cleveland, Ohio}

The $\mathrm{C}$ language is well suited for the low-level operations needed in the programming of real-time experiments on small computers. However, because $\mathbf{C}$ is designed to be nonspecific to a particular machine or operating system, it does not itself contain primitive commands for controlling special input and output devices, nor for timing. Fortunately, all popular MS-DOS implementations of C include procedures for calling the many predefined routines that are part of the operating system and that are available to user programs.

The MS-DOS function that is most useful for timing the durations of events is int $21 \mathrm{~h}$, function $2 \mathrm{ch}$, which is documented in MS-DOS technical reference manuals. Function $2 \mathrm{ch}$ returns four binary bytes of information in the CPU C and D registers, giving the elapsed time in hours, minutes, seconds, and hundredths of a second. Out of half a dozen inexpensive $C$ implementations that $I$ have examined, only Datalight $\mathrm{C}$ furnishes a predefined tier function using int $21 \mathrm{~h}$, function $2 \mathrm{ch}$. The $\mathrm{C}$ version coded in Listing 1 is named clock( ), as was Datalight's original version. Using the same name enhances portability of source programs that employ this timing method. However, Datalight's version will not compile with most other compilers, so the version in Listing 1 is designed to perform the same as Datalight's but to compile with Microsoft $\mathbf{C}$ and Borland Turbo $\mathrm{C}$. It is somewhat unusual to find different $C$ compilers that access the operating system functions in exactly the same way. Users of compilers other than these two may need to consult their documentation and change several lines of code to get this clock() routine to compile properly. In Listing 2, a test program that merely times intervals between successive presses of the ENTER key is shown. Only the ENTER key should be used with this simple test program, because getchar() input is line-buffered with most implementations of $\mathbf{C}$.

The clock() routine returns a long integer (32 bits) that is the elapsed time since midnight, in units of $.01 \mathrm{sec}$. It reads the registers with which MS-DOS maintains its time-of-day clock. Clock() does have some limitations. It provides no way to reset the counter to zero, so durations must be timed by taking the differences between successive readings. A difference between readings that straddle midnight on MS-DOS's time-of-day clock will be incorrect, because MS-DOS resets the counter to zero every 24 hours. Moreover, the true resolution is gener-

The author's mailing address is: Department of Psychology, Cleveland State University, Cleveland, $\mathrm{OH} \mathbf{4 4 1 1 5 .}$
LSTING 1

The Clock() Routine Returning the Elapsed Time Since Midnight in Units of $.01 \mathrm{Sec}$

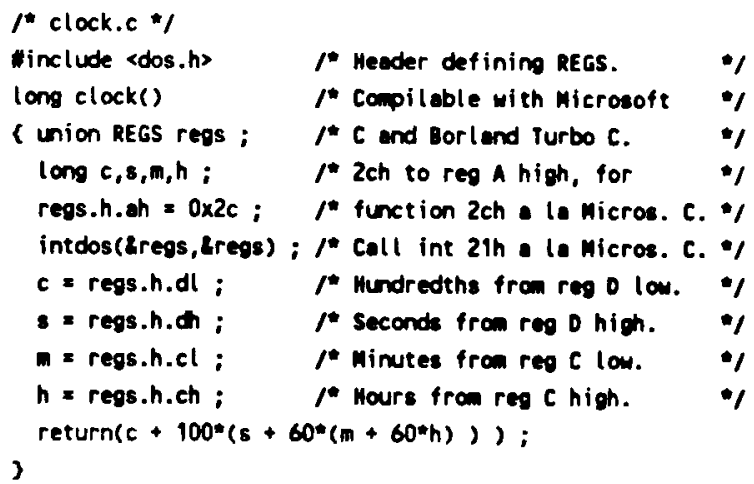

LISTING 2

A Test Program Giving The Times Between Successive Presses of the ENTER Key

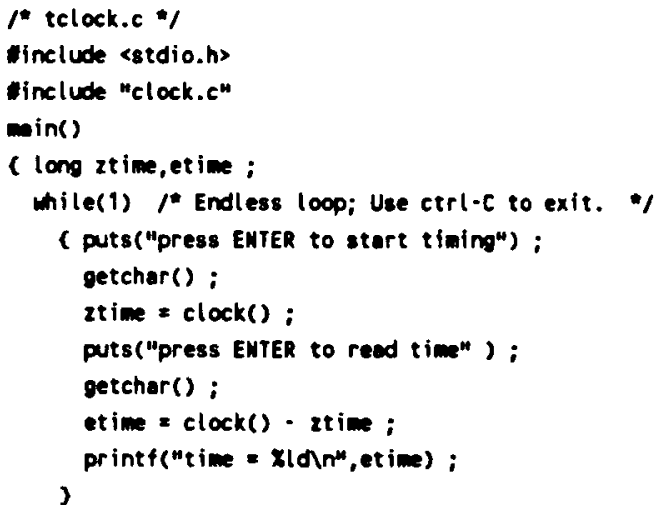

ally not $.01 \mathrm{sec}$, and it is considerably worse on most machines. On the IBM PC and PS/2 series the resolution is only about $.055 \mathrm{sec}$. On the 8-MHz Slicer, it is .02 sec.'

Offsetting these limitations are several advantages. The clock() routine is small, and it gives times in the same units of $.01 \mathrm{sec}$ even on other machines that have different timing resolutions and CPU speeds. It does not require that any special equipment be installed, and it does not interfere with other processes that make normal use of the MS-DOS time-of-day clock. It consumes no updating time of its own, because MS-DOS does the updating. It does not intercept the MS-DOS clock interrupts, so there is no need to restore the interrupt vector after use. The interrupts that MS-DOS uses to update its timeof-day clock are of the highest priority, above those used for the keyboard, disks, printers, and serial interfaces. Thus, there is no risk that any normal input or output operation will disturb the counter that is accessed by clock( ). In short, $\operatorname{clock}()$ is ideal in most ways apart from its crude timing resolution. ${ }^{2}$ 
There are other MS-DOS C-language timing methods available for cases where greater resolution is needed, such as one that uses the timing capabilities of the serial interface of an IBM PC (Emerson, 1988). However, none combines the simplicity, reliability, and unobtrusiveness of this one. ${ }^{3}$

\section{REFERENCE}

Emerson, P. L. (1988). Using serial interfaces and the C language for real-time experiments. Behavior Research Methods, Instruments, \& Computers, 20, 330-336.

\section{NOTES}

1. When MS-DOS is installed on a non-IBM computer, some of the internal constants are usually adjusted in order to make the operating system behave in essentially the same way as on the IBM PC, even though the hardware clock speed is different and a different BIOS is used.

2. A reviewer suggested a technique that reduces timing uncertainty for computer initiated intervals. It is to have the program wait before beginning the interval to be timed, until just after the clock count has changed. On an IBM PC, for example, this would roughly halve the timing uncertainty range from about 110 to $55 \mathrm{msec}$.

3. Potential users who have any need for soft copy may write the author, who will supply the source code on a 5.25 in. diskette in IBM $360 \mathrm{~K}$ format.

(Revision accepted for publication August 2, 1988.) 